\title{
Significance of the progesterone receptor and epidermal growth factor receptor, but not the estrogen receptor, in chemically induced lung carcinogenesis in female $\mathrm{A} / \mathrm{J}$ mice
}

\author{
SOSUKE KISHI $^{1}$, MASANAO YOKOHIRA ${ }^{1}$, KEIKO YAMAKAWA ${ }^{1}$, \\ KOUSUKE SAOO $^{1,2}$ and KATSUMI IMAIDA ${ }^{1}$ \\ ${ }^{1}$ Department of Pathology and Host-Defense, Faculty of Medicine, Kagawa University, Kagawa 761-0793; \\ ${ }^{2}$ Department of Diagnostic Pathology, Tomakomai City Hospital, Hokkaido 053-0034, Japan
}

Received October 15, 2013; Accepted July 8, 2014

DOI: $10.3892 /$ ol.2014.2559

\begin{abstract}
In the present study, the expression levels of female hormone receptors, estrogen receptor (ER) and progesterone receptor (PR) and the epidermal growth factor receptor, (EGFR), as well as proliferating cell nuclear antigen (PCNA) were examined in lung tumors that were induced by 4-(methylnitrosamino)-1-(3-pyridyl)-1-butanone (NNK) in female A/J mice. Each seven-week-old mouse was administered with $2 \mathrm{mg}$ NNK via intraperitoneal injection and the mice were subsequently euthanized at week 52. Lung tumors, including adenomas, carcinomas in adenomas and adenocarcinomas, were obtained and analyzed by immunohistochemistry for the expression levels of the receptors, ER, PR and EGFR, and PCNA. The results were as follows: i) In mouse lung adenomas, a significant correlation was identified between the size of the tumor and PCNA expression, although not with the expression of the receptors (ER, PR and EGFR); ii) in the carcinoma components of the carcinomas in adenomas, the size of the tumor and PCNA expression were correlated, while EGFR expression demonstrated a significant correlation with PR expression; iii) in adenocarcinomas, the tumor size significantly correlated with PCNA, EGFR and PR expression; and iv) EGFR and PR expression was identified to be significantly correlated in adenocarcinomas, and to a certain extent in the carcinoma components of the carcinomas in adenomas, although not in the adenomas. Notably, ER expression was not associated with tumor growth or the other factors, particularly EGFR expression, and no significant differences were identified between the
\end{abstract}

Correspondence to: Professor Katsumi Imaida, Department of Pathology and Host-Defense, Faculty of Medicine, Kagawa University, 1750-1 Ikenobe, Miki-cho, Kita-gun, Kagawa 761-0793, Japan

E-mail: imaida@med.kagawa-u.ac.jp

Key words: lung carcinogenesis, progesterone receptor, estrogen receptor, epidermal growth factor receptor, non-small cell lung cancer three types of lesion. These results indicate that PR, like EGFR, may be significant in lung carcinogenesis.

\section{Introduction}

Lung cancer is the most common cause of cancer-associated mortality in males and females worldwide (1). Non-small cell lung cancer (NSCLC) is the predominant type of lung cancer, including the major histologic types, such as adeno-, squamous cell and large cell carcinoma. In addition, advanced NSCLC is the most common cause of cancer-associated mortality in males and females in the United States (2). Furthermore, a large number of individuals globally suffer from NSCLC, thus, novel treatment and prevention approaches for this disease are urgently required.

Despite a decline in the smoking population, the incidence of NSCLC, particularly the adenocarcinoma subtype, continues to increase $(3,4)$. Adenocarcinoma is the most frequent histologic type of NSCLC in non-smokers and young patients $(4,5)$. This indicates that etiologic factors other than tobacco may be involved in the development and progression of NSCLC.

Numerous studies have reported that female sex hormones, particularly endogenous and exogenous estrogens, may contribute to NSCLC in females (6-9), and estrogen receptors (ERs) are expressed in NSCLC tissue and may be associated with neoplasia (6-11). Nuclear and extranuclear ERs appear to act concomitantly to promote cell growth $(12,13)$.

In the normal lung tissue of humans and rodents, ERs perform important physiological functions to promote proliferation, including those that are required for alveolar formation in the development, alveolar regeneration and maintenance of the pulmonary diffusion capacity $(14,15)$.

The progesterone receptor (PR) mediates the effect of progestins and it has been reported that the progression of spontaneous lung tumors was reduced by treatment with antiprogestin (Mifepristone, also termed RU-486) in mice (16). However, the association between the intensity of PR expression and the biological characteristics of lung cancer tissue remains unclear. Furthermore, PR expression in human lung tumors varies markedly, from a high expression frequency of $39-63 \%$ (17-19) to marginal (22-35\%) or no expression $(20,21)$. 
It has previously been reported that the subset of patients with NSCLC, predominantly those with adenocarcinomas, possess a specific activating mutation in the epidermal growth factor receptor (EGFR) gene, which correlates with marked clinical responsiveness to the EGFR tyrosine kinase inhibitors, gefitinib or erlotinib (22-24). This was subsequently identified by studies, which consistently demonstrated that mutations in the tyrosine kinase domain of EGFR are more commonly found among females, never-smokers, Asian individuals and those exhibiting adenocarcinomas (25-30). However, despite an almost universal presence of EGFR in NSCLC tumors, the therapeutic inhibition of EGFR by gefitinib and erlotinib results in significant tumor regression in only $10-20 \%$ of patients, again indicating the variation in the molecular pathogenesis of the different types of lung cancer.

The primary causes of NSCLC, not including tobacco smoking, remain unclear. Although sex hormones have been implicated as a cause of lung cancer in non-smoking females there are numerous additional unidentified mechanisms. The aim of the present study was to elucidate the pathognomonic factors that promote the progression from adenoma to adenocarcinoma.

Rodent models of lung carcinogenesis are effective tools for investigating the underlying mechanisms of human NSCLC development and for the detection of chemopreventive agents, as the morphologies, histogenesis and molecular characteristics of induced primary rodent lung tumors are similar to those of humans. The A/J mouse, which demonstrates increased spontaneous lung tumor development in the females than in the males, was selected for the present study. In addition, the female $\mathrm{A} / \mathrm{J}$ mouse is particularly sensitive to lung carcinogenesis induced by 4-(methylnitrosamino)-1-(3-pyridyl)-1-butanone (NNK), a tobacco-specific nitrosamine component of tobacco smoke (31). KRAS mutations are frequently observed in this type of rodent lung carcinogenesis model, which was also noted in a case of human NSCLC (32).

In the present study, the expression of female hormone receptors (ER and PR), as well as EGFR and PCNA, was examined in NNK-induced lung tumors in female $\mathrm{A} / \mathrm{J}$ mice (a rodent lung carcinogenesis model) in order to investigate the possible associations between different factors in mouse lung tumors, including adenomas, carcinomas in adenomas and adenocarcinomas.

\section{Materials and methods}

Chemicals. NNK was purchased from Toronto Research Chemicals Inc. (Toronto, Canada).

Animals. Female A/J mice (age, 5 weeks), purchased from Shizuoka Laboratory Animal Center (Shizuoka, Japan) were maintained in the Division of Animal Experiments, Life Science Research Center, Kagawa University (Kagawa, Japan), in accordance with the Institutional Regulations for Animal Experiments. These regulations include the best considerations for animal welfare and good practice of animal handling, contributing to the replacement, refinement and reduction of animal testing. The protocol of the experiment was approved by the Animal Care and use Committee for Kagawa University. The animals were housed in polycarbonate cages with re-used paper chips (EchoChip ${ }^{\circledR}$, CL-4163; CLEA Japan, Inc., Tokyo, Japan) for bedding, and given free access to drinking water and a basal diet (Oriental MF; Oriental Yeast Co., Ltd., Tokyo, Japan). The conditions were controlled as follows: Humidity, $60 \pm 10 \%$; lighting, 12-h light/dark cycle; and temperature, $24 \pm 2^{\circ} \mathrm{C}$. The experiments commenced following a 2-week acclimatization period.

Experimental design and tissue preparation. Details of the original experiment were published by Takeuchi et al (33) and the tissue samples from one group in the experiment were used in the present study. Briefly, at the beginning of the current experiment, 20 mice (age, 7 weeks) were each administered with a single dose of NNK $(2 \mathrm{mg} / 0.1 \mathrm{ml}$ saline $)$ via intraperitoneal injection. The mice did not receive any further treatment. The experiment was terminated at 52 weeks, when all of the surviving mice were euthanized under deep anesthesia, and their lungs were excised and weighed. The final weights of the lungs were calculated by subtracting the weights of the trachea and heart. The lungs were subsequently infused with $10 \%$ neutral-buffered formalin (Wako Pure Chemical Industries, Ltd., Osaka, Japan) and inspected grossly. In addition, the macroscopically detected lung nodules were counted under a stereomicroscope (Olympus SZ microscope; Olympus, Tokyo, Japan) and were routinely processed for embedding in paraffin. Histopathological examination with hematoxylin and eosin (H\&E) was conducted by staining each lung lobe section. Proliferative lesions of the lung were diagnosed as hyperplasia, adenoma or adenocarcinoma according to the International Harmonization of Nomenclature and Diagnostic Criteria (INHAND) (34). Due to differences in terminology used in INHAND, 'hyperplasia, bronchiolo-alveolar', 'adenoma, bronchiolo-alveolar' and 'adenocarcinoma, bronchiolo-alveolar' were abbreviated to hyperplasia, adenoma and adenocarcinoma, respectively, in the present study.

In the H\&E-stained slides, the number of lung proliferative lesions was counted and the major axis size of the lung tumors was measured under an Olympus BX51 microscope with an Olympus DP 70 digital camera, using an Olympus DP controller and the Olympus DP manager software (all Olympus).

To avoid errors and repeat counting, lung maps were prepared by transcribing all of the H\&E-stained slides onto paper, and the tumor locations and histopathologic types were marked on each lung map during the microscopic investigation.

Immunohistochemical analysis. The lung tissues were immunostained for EGFR, ER, PR and PCNA using the avidin-biotin complex method (Ventana Medical Systems; Oro Valley, AZ, USA); all of the processes, from deparaffinization to counterstaining with hematoxylin, were performed automatically using the Ventana Discovery ${ }^{\mathrm{TM}}$ staining system (Ventana Medical Systems). The primary rabbit anti-human EGFR polyclonal antibody, EGFR(1005), sc-03; anti-human ER $\alpha$ rabbit polyclonal antibody, $\mathrm{ER} \alpha(\mathrm{H}-184)$, sc-7207; and anti-human PR rabbit polyclonal antibody and PR(CR-19), sc-538 were purchased from Santa Cruz Biotechnology, Inc. (Santa Cruz, CA, USA) and were all used at a dilution of 1:50. Anti-human PCNA goat polyclonal antibody, PCNA(c-20), sc-9857 (Santa Cruz Biotechnology, Inc.) was used at a dilution of 1:400. The secondary antibodies used were biotinylated goat anti-rabbit 


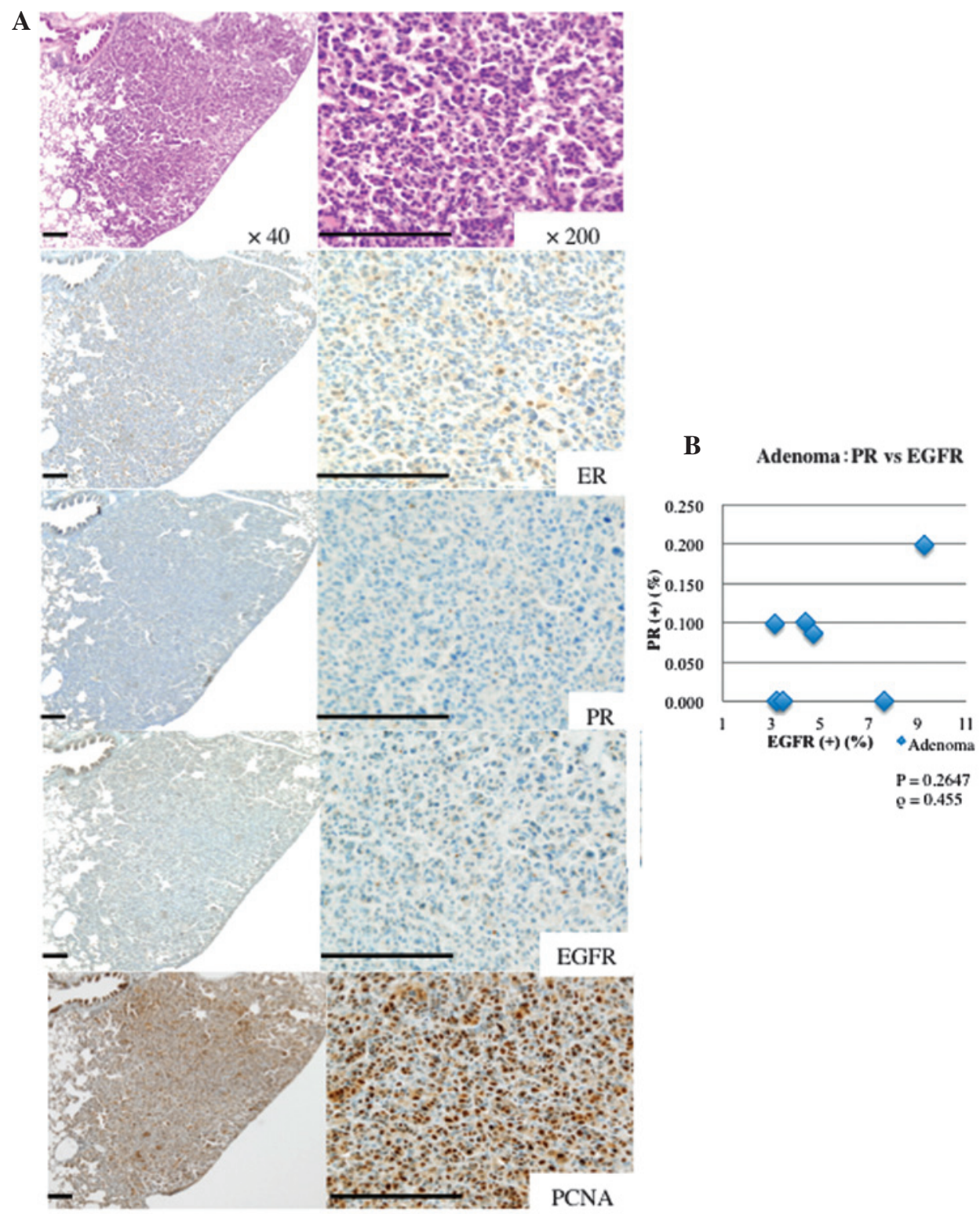

Figure 1. Histology and immunohistochemical findings for adenomas. (A) Histochemical and immunohistochemical findings of an adenoma (hematoxylin and eosin staining for ER, PR, EGFR and PCNA). (B) There was no correlation between the PR and EGFR labeling indices in adenomas. ER, estrogen receptor; PR, progesterone receptor; EGFR, epidermal growth factor receptor; PCNA, proliferating cell nuclear antigen.

IgG (BA-1000; Vector Laboratories, Inc., Burlingame, CA, USA) and biotinylated rabbit anti-goat IgG (BA-5000, Vector Laboratories, Inc.). The sections were incubated with the primary antibody (antibodies for ER, PR, and EGFR diluted at 1:50 and diluted at 1:400 for PCNA) for $12 \mathrm{~h}$ at $4^{\circ} \mathrm{C}$ and were incubated with the secondary antibody for $30 \mathrm{~min}$.

The labeling indices were carefully assessed, to identify the cellular-positive areas (cellular membrane-, cytoplasm- or nucleus-positivity for each antibody in the tumor cells) whilst avoiding duplicate counting of the same tumor cells. Cells were considered to be positive when ER, PR, and PCNA staining was observed in the nuclei and when EGFR staining was noted on the cellular membranes.

The protocol for counting the labeling indices involved the following five steps to ensure accuracy: i) Drawing outlines of the histological H\&E-stained slides of each lung tissue macroscopically on paper, termed lung maps; ii) marking the tumor locations and writing the histopathologic type on the lung map; iii) randomly selecting typical areas of each lung tumor, using an Olympus BX51 microscope equipped with an Olympus UPlanSApo 20x/0.75 objective lens (Olympus), and obtaining at least three images per single lung tumor without overlap using an Olympus DP 70 (Olympus) and Olympus DP controller and manager software; iv) printing all color images on A4 paper (210x297 mm) with a laser color printer, allowing counts of $>1,000$ cells per tumor lesion from three color copied sheets; v) counting the positive cells and putting a red tick mark on the first count and subsequently counting the negative cells with pencil marks on the second count.

Statistical analysis. Correlations between the expression levels of EGFR, ER, PR, PCNA and the size of lung proliferative lesions were analyzed by Spearman's rank test. $\mathrm{P}<0.05$ was considered to indicate a statistically significant difference. 
A
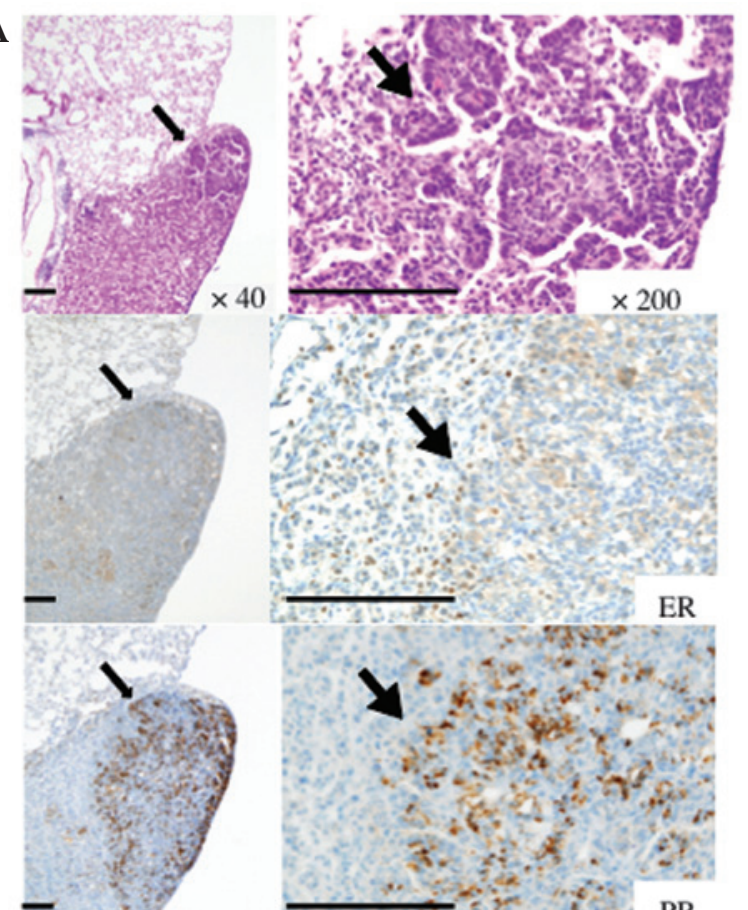

B Carcinoma in adenoma :PR vs EGFR

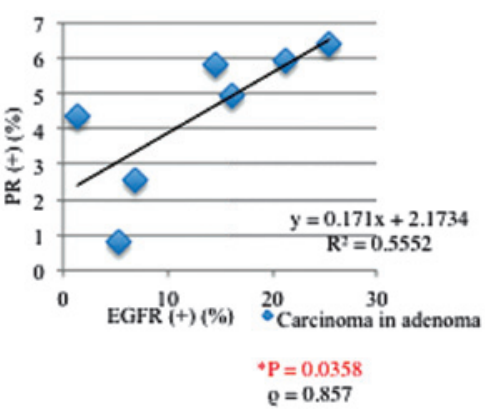

Figure 2. (A) Histochemical and immunohistochemical findings of carcinoma in adenoma (hematoxylin and eosin staining for ER, PR, EGFR and PCNA). Strong expression of PR, EGFR and PCNA was observed in the carcinoma component, although not in the peripheral adenoma component (black arrows). (B) A significant correlation $(\mathrm{P}<0.05)$ was observed between the PR and EGFR labeling indices of the carcinoma components of the carcinomas in adenomas. $\mathrm{ER}$, estrogen receptor; PR, progesterone receptor; EGFR, epidermal growth factor receptor; PCNA, proliferating cell nuclear antigen.

Multiple comparisons between the labeling indices and the tumor size for the three histopathological types of lung tumors were conducted using the Tukey-Kramer test.

\section{Results}

Selection of lung tumor samples. Initially, there were 20 mice included in the study, however, one succumbed during the experiment and was not included in the effective numbers. Lung proliferative lesions, hyperplasia, adenoma and adenocarcinoma were diagnosed according to the criteria in the INHAND (34). Certain lung adenocarcinomas were identified within adenomas, therefore, these lesions were diagnosed as carcinomas in adenomas. All 19 animals exhibited adenomas (incidence, $100 \%$ and multiplicity, $7.79 \pm 3.44 /$ mouse) and the total malignant tumors (a combination of lung adenocarcinomas and carcinoma in adenoma) were counted in 18/19 mice (incidence, $94.7 \%$ and multiplicity, $4.68 \pm 3.42 /$ mouse). The carcinomas in adenomas accounted for $61.8 \%$ of the total malignant tumors and the remaining $38.2 \%$ were adenocarcinomas.

Tumor characteristics. The investigated lung tumors, which could be observed in the H\&E-stained slides as well as in all of the immunohistochemically-stained slides, were selected for evaluation. Samples were insufficient for staining in two mice due to their size, therefore, out of the remaining 17 mice, seven adenomas, seven carcinomas in adenomas and 17 carcinomas were selected. Only the carcinoma components were analyzed from the carcinomas in adenoma samples.

The histopathological and immunohistochemical findings from the three histopathological types of lung tumor nodules 
A
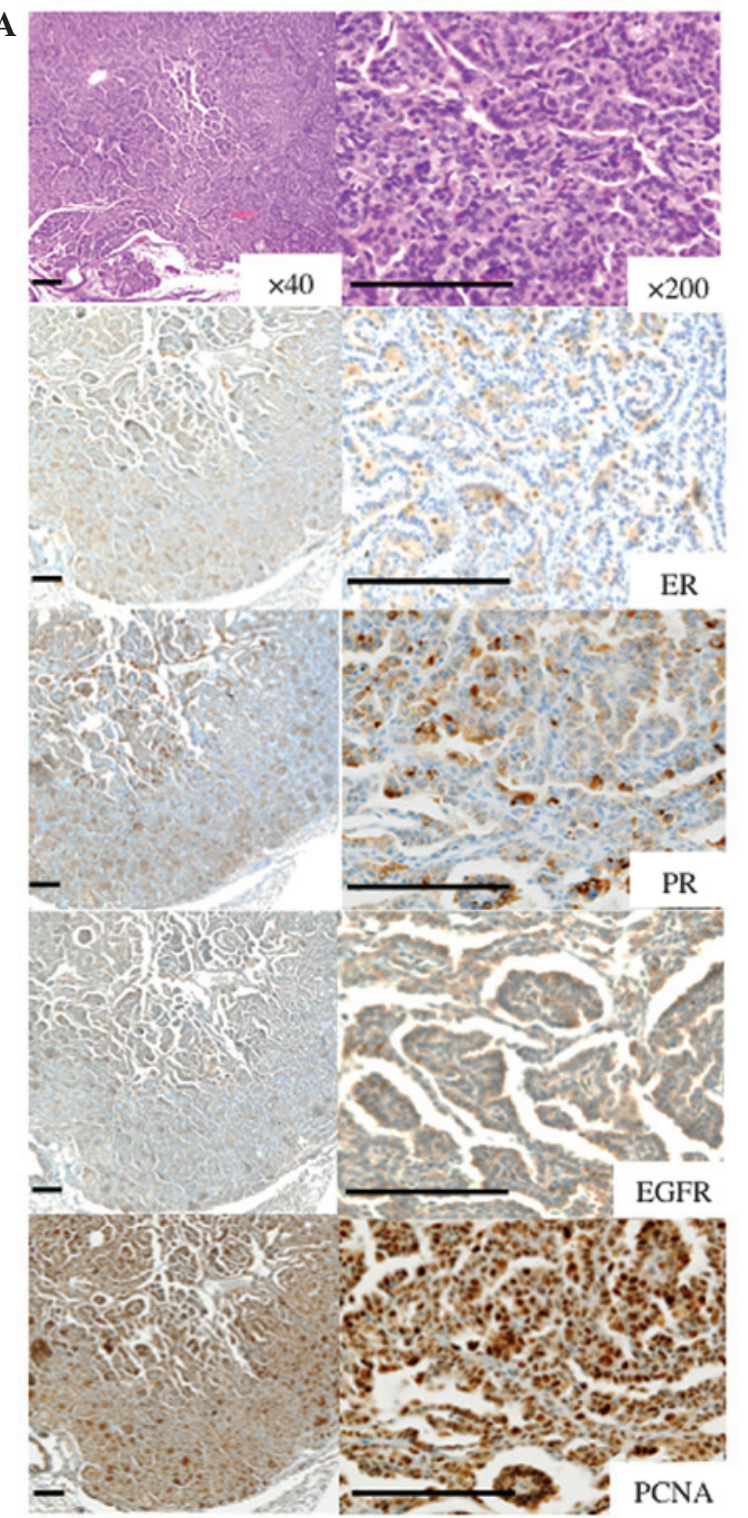

B Adenocarcinoma: PR vs EGFR

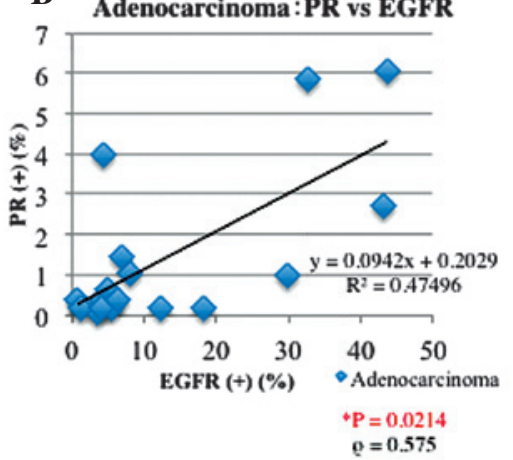

Figure 3. (A) Histology and immunohistochemical findings for adenocarcinomas (hematoxylin and eosin staining for ER, PR, EGFR and PCNA). (B) A significant correlation $(\mathrm{P}<0.05)$ between the PR and EGFR labeling indices was noted in the adenocarcinomas. ER, estrogen receptor; PR, progesterone receptor; EGFR, epidermal growth factor receptor; PCNA, proliferating cell nuclear antigen.

(adenomas, carcinomas in adenoma and adenocarcinomas) are summarized in Figs. 1-3. A strong expression of PR, EGFR and PCNA was observed in the carcinoma component of carcinomas in adenomas, in contrast to the peripheral adenoma component (Fig. 2). The major axis size of lung tumors on the H\&E-stained slides was measured under a microscope. Data for the tumor size, and the labeling indices of the ER, PR, EGFR and PCNA-positive cells in each tumor type are summarized in Table I. Although, the adenomas tended to be larger than the other types of tumors, all of the immunohistochemical labeling indices (PCNA, ER, PR and EGFR) tended to be lower when compared with the carcinomas in the adenomas and adenocarcinoma groups. (Turkey-Kramer test: $\mathrm{PCNA}, \mathrm{P}<0.01$ and $\mathrm{PR}, \mathrm{P}<0.001$; Table I). Although PR-positive tumor cells were observed in the carcinomas in adenomas and in the adenocarcinomas, the adenomas were generally PR-negative. However, ER expression was similar in all of the histopathologic types.
Using statistical analysis, correlation between five factors (the expression of EGFR in the cellular membranes, the expression of ER, PR, and PCNA in the nucleus and the size of the tumor [Fig. 4]) was detected using nonparametric correlation analysis (Spearman's rank correlation) on 10 combined paired comparisons $\left({ }_{5} C_{2}=10\right)$. The only statistically significant correlation between tumor size and PCNA expression was observed in the adenomas. Similarly, the carcinoma components of carcinomas in adenomas demonstrated significant correlations between tumor size and PCNA expression, as well as between EGFR and PR expression. No significant correlation between the size of the tumor and PCNA expression was observed in the adenocarcinomas. By contrast, significant correlations were identified between tumor size and EGFR expression and tumor size and PR expression. Furthermore, EGFR expression demonstrated a significant correlation with PR expression, as was also observed in the carcinoma components in carcinomas 
Table I. Tumor size and immunohistochemical labeling indices for the positive cells in each tumor type ${ }^{\mathrm{a}}$.

\begin{tabular}{lccc}
\hline Variable & Adenoma & Carcinoma in adenoma $^{\mathrm{c}}$ & Adenocarcinom $^{\circ}$ \\
\hline Tumors, $\mathrm{n}$ & 7 & 7 & 17 \\
Tumor size, $\mathrm{mm}$ & $1.9 \pm 0.4$ & $0.6 \pm 0.3^{\mathrm{d}}$ & $1.8 \pm 0.7$ \\
Labeling indices, $\%$ & & & \\
PCNA & $60.7 \pm 13.0$ & $84.1 \pm 5.4^{\mathrm{e}}$ & $71.8 \pm 12.6$ \\
EGFR $^{\mathrm{b}}$ & $5.1 \pm 2.4$ & $13.0 \pm 8.7$ & $13.5 \pm 14.5$ \\
ER $^{\mathrm{b}}$ & $6.4 \pm 3.1$ & $6.7 \pm 3.2$ & $6.7 \pm 4.5$ \\
PR $^{\mathrm{b}}$ & $0.1 \pm 0.1$ & $4.4 \pm 2.0^{\mathrm{d}}$ & $1.5 \pm 2.0$
\end{tabular}

${ }^{a}$ Values are expressed as the mean \pm standard deviation; ${ }^{b}$ expressed as nuclear-positive; ${ }^{\text {conly }}$ the carcinoma components were evaluated; ${ }^{\mathrm{d}}$ significantly different compared with adenomas $(\mathrm{P}<0.001$; Turkey-Krammer test $)$; ${ }^{\text {e }}$ significantly different compared with adenomas $(\mathrm{P}<0.01$; Turkey-Krammer test). PCNA, proliferating cell nuclear antigen; EGFR, epidermal growth factor receptor; ER, estrogen receptor; PR, progesterone receptor.

Adenoma
\begin{tabular}{|l|l|l|l|l|l|}
\hline & Tumor size & PCNA $^{\mathrm{b}}$ & EGFR $^{\mathrm{c}}$ & $\mathrm{ER}^{\mathrm{b}}$ & $\mathrm{PR}^{\mathrm{b}}$ \\
\hline Tumor size & & & & & \\
\hline PCNA & $\mathrm{a}^{\text {"1 }}$ & & & & \\
\hline EGFR & & & & & \\
\hline ER & & & & & \\
\hline PR & & & & & \\
\hline
\end{tabular}

Carcinoma in adenoma ${ }^{\mathrm{d}}$
\begin{tabular}{|l|c|l|l|l|l|}
\hline & Tumor size & PCNA & EGFR & ER & PR \\
\hline Tumor size & & & & & \\
\hline PCNA & $\mathrm{a}^{* 1}$ & & & & \\
\hline EGFR & & & & & \\
\hline ER & & & & & \\
\hline PR & & & $\mathrm{a}^{1 / 2}$ & & \\
\hline
\end{tabular}

Adenocarcinoma
\begin{tabular}{|l|c|l|l|l|l|}
\hline & Tumor size & PCNA & EGFR & ER & PR \\
\hline Tumor size & & & & & \\
\hline PCNA & & & & & \\
\hline EGFR & $\mathrm{a}^{4 / 3}$ & $\mathrm{a}^{1 / 3}$ & & & \\
\hline ER & & & & & \\
\hline PR & $\mathrm{a}^{4 / 3}$ & & $\mathrm{a}^{4 / 2}$ & & \\
\hline
\end{tabular}

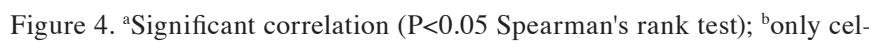
lular membrane expression was evaluated; 'only carcinoma components of the carcinoma in adenoma were evaluated; ${ }^{\# 1}$ significant correlation between the size of the tumor vs. the PCNA expression in the adenoma and the carcinoma in adenoma. Blue coloring signifies that the same tendency was observed in the adenoma and the carcinoma component of the carcinoma in adenoma; ${ }^{\# 2}$ significant correlation identified between PR vs. EGFR expression in the carcinoma in adenoma and the adenocarcinoma. Yellow coloring signifies that the same tendency was observed in the carcinoma component of the carcinoma in adenoma and the adenomcarcinoma; ${ }^{\# 3}$ in just adenocarcnima, significant correlation was identified between the size of the tumor vs. EGFR, the size of the tumor vs. PR and PCNA vs. EGFR expression. Red coloring signifies that the significant associations were only observerd in the adenocarcinoma. PCNA, proliferating cell nuclear antigen; EGFR, epidermal growth factor receptor; ER, estrogen receptor; $\mathrm{PR}$, progesterone receptor.

in adenomas. In addition, ER was not identified to be correlated with the other factors and no significant differences were noted between the three histopathological types. These findings are summarized in a schematic illustration in Fig. 5.

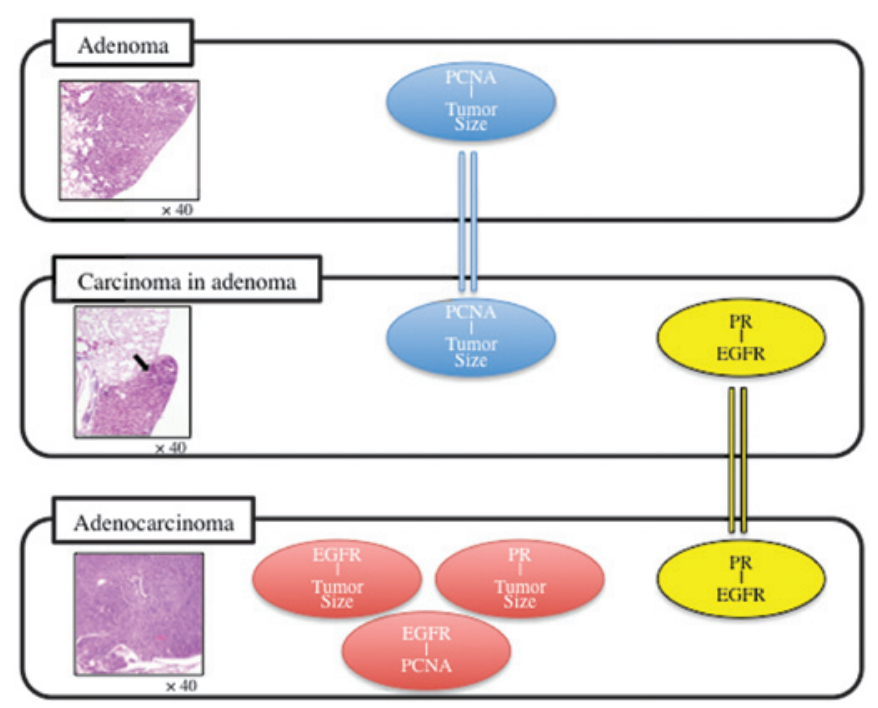

Figure 5. Schematic of the immunohistochemical findings, demonstrating the significant correlation between each histopathological type and the correlating factors within each of the three histopathological types. PCNA, proliferating cell nuclear antigen; PR, progesterone receptor; EGFR, epidermal growth factor receptor.

\section{Discussion}

The present study demonstrated significant correlations between tumor size and the EGFR-positive indices, as well as between EGFR and PR expression in adenocarcinomas and the carcinoma components of carcinomas in adenomas. This indicates that PR and EGFR may have significant roles in tumor progression during NNK-induced mouse lung carcinogenesis. By contrast, ER expression was not identified to correlate with EGFR expression or tumor size; therefore, ER and PR may differ with regard to their influence on lung carcinogenesis.

A randomized prospective human study, as part of a multi-component clinical trial (the Coronary Drug Project) identified males who had previously suffered from a myocardial infarction. The aim was to evaluate the difference between the effects of administering $2.5 \mathrm{mg} /$ day estrogen or a placebo; anticipating a reduction in future cardiac events. However, 
it was discontinued as a result of an increased incidence of lung cancer-associated mortality, which was observed in the estrogen treatment group (35). In addition, two randomized prospective trials proposed that hormone replacement therapy with estrogen plus progestins increased the incidence of and mortality from lung cancer in postmenopausal females $(36,37)$. However, similar research with estrogen alone did not indicate increased lung cancer-associated mortality in females (38).

In mice, one study reported that the progression of spontaneous lung tumors was reduced with treatment using antiprogestin (Mifepristone; RU-486) (16).

Previously, mutations in codon 12 of the KRAS gene were detected in carcinogen-induced rodent lung tumor models (31), with similar incidences noted to those that were previously reported during an investigation of X-ray-induced rat lung tumors (39). Various molecular studies regarding human lung cancer indicate that the notable differences in p53 and KRAS mutations are between the non-smokers and smokers. Overall, KRAS mutations have been identified to be more frequent in smokers compared with in non-smokers; although, those in codon 12 of the KRAS gene $(\mathrm{G} \rightarrow \mathrm{T}$ transversions and $\mathrm{G} \rightarrow \mathrm{A}$ transitions) are found in non-smokers and smokers (41). Conversely, although the frequency of mutation in the p53 gene was similar in non-smokers when compared with smokers, the types and spectra of mutations were identified to be significantly different. Furthermore, $\mathrm{C} \rightarrow \mathrm{T}$ transitions are found only in non-smokers $(40,41)$. The frequency of the EGFR mutation in human NSCLC is relatively high (26-40\% in Asian patients and 2-12\% in non-Asian patients). In one animal model, development of urethane-induced lung adenomas in male A/J mice was inhibited by an EGFR-tyrosine kinase (TK) domain inhibitor, despite a lack of any mutation in the EGFR-TK domain, which was proposed to be due to activated EGFR signaling (42). Thus, activation of the EGFR signaling pathway, caused by genetic mechanisms without somatic mutations of EGFR, may be involved in the growth of lung tumors in humans and animals. All the available data indicates that the variety and pathogenesis of NSCLC in never-smokers may be different compared with in smokers, with the involvement of additional factors considered to be significant.

In conclusion, this study demonstrated a significant correlations between tumor size and the EGFR-positive indices, as well as between EGFR and PR expression in adenocarcinomas and the carcinoma components of carcinomas in adenomas. This indicates that PR and EGFR may exhibit significant roles in tumor progression during NNK-induced mouse lung carcinogenesis. In order to study the participation of etiological factors in NSCLC progression, further studies are required to focus on the correlations between sex hormone functions and other growth factors in the sequential genesis of malignancies.

\section{Acknowledgements}

The authors would like to thank Dr Yoko Matsuda (Integrative Oncological Pathology, Nippon Medical School, Tokyo, Japan) for expert advice on this study and
Dr Malcolm A. Moore for help with the preparation and critical reading of this article.

\section{References}

1. Ferlay J, Shin HR, Bray F, et al: Estimates of worldwide burden of cancer in 2008: GLOBOCAN 2008. Int J Cancer 127: 2893-2917, 2010.

2. Jemal A, Murray T, Ward E, et al: Cancer statistics, 2005. CA Cancer J Clin 55: 10-30, 2005

3. Patel JD, Bach PB and Kris MG: Lung cancer in US women: a contempory epidemic. JAMA 291: 1763-1768, 2004.

4. Jemal A, Ward E and Thun M: Declining death rates reflect progress against cancer. PLoS One 5: e9584, 2010.

5. Subramanian J and Govindan R: Lung cancer in never smokers: a review. J Clin Oncol 25: 561-570, 2007.

6. Stabile LP, Davis AL, Gubish CT, et al: Human non-small cell lung tumors and cells derived from normal lung express both estrogen receptor alpha and beta and show biological responses to estrogen. Cancer Res 62: 2141-2150, 2002.

7. Niikawa H, Suzuki T, Miki Y, et al: Intratumoral estrogens and estrogen receptors in human non-small cell lung carcinoma. Clin Cancer Res 14: 4417-4426, 2008.

8. Taioli E and Wynder EL: Re: Endocrine factors and adenocarcinoma of the lung in women. J Natl Cancer Inst 86: 869-870, 1994.

9. Ganti AK, Sahmoun AE, Panwalkar AW, Tendulkar KK and Potti A: Hormone replacement therapy is associated with decreased survival in women with lung cancer. J Clin Oncol 24: 59-63, 2006

10. Weinberg OK, Marquez-Garban DC, Fishbein MC, et al: Aromatase inhibitors in human lung cancer therapy. Cancer Res 65: 11287-11291, 2005

11. Pietras RJ, Márquez DC, Chen HW, et al: Estrogen and growth factor receptor interactions in human breast and non-small cell lung cancer cells. Steroids 70: 372-381, 2005.

12. Pietras RJ and Márquez-Garbán DC: Membrane-associated estrogen receptor signaling pathways in human cancers. Clin Cancer Res 13: 4672-4676, 2007.

13. Gruber CJ, Tschugguel W, Schneeberger C and Huber JC: Production and actions of estrogens. N Engl J Med 346: 340-352, 2002.

14. Massaro D, Clerch LB and Massaro GD: Estrogen receptor-alpha regulates pulmonary alveolar loss and regeneration in female mice: morphometric and gene expression studies. Am J Physiol Lung Cell Mol Physiol 293: L222-L228, 2007.

15. Morani A, Warner M and Gustafsson JA: Biological functions and clinical implications of oestrogen receptors alfa and beta in epithelial tissues. J Intern Med 264: 128-142, 2008.

16. Check JH, Sansoucie L, Chern J and Dix E: Mifepristone treatment improves length and quality of survival of mice with spontaneous lung cancer. Anticancer Res 30: 119-122, 2010.

17. Raso MG, Behrens C, Herynk MH, et al: Immunohistochemical expression of estrogen and progesterone receptors identifies a subset of NSCLCs and correlates with EGFR mutation. Clin Cancer Res 15: 5359-5368, 2009.

18. Su J-M, Hsu HK, Chang H, et al: Expression of estrogen and progesterone receptors in non-small-cell lung cancer: immunohistochemical study. Anticancer Res 16: 3803-3806, 1996.

19. Stabile LP, Dacic S, Land SR, et al: Combined analysis of estrogen receptor beta-1 and progesterone receptor expression identifies lung cancer patients with poor outcome. Clin Cancer Res 17: 154-164, 2011.

20. Skov BG, Fischer BM and Pappot H: Oestrogen receptor beta over expression in males with non-small cell lung cancer is associated with better survival. Lung Cancer 59: 88-94, 2008.

21. Di Nunno L, Larsson LG, Rinehart JJ and Beissner RS: Estrogen and progesterone receptors in non-small cell lung cancer in 248 consecutive patients who underwent surgical resection. Arch Pathol Lab Med 124: 1467-1470, 2000.

22. Lynch TJ, Bell DW, Sordella R, et al: Activating mutations in the epidermal growth factor receptor underlying responsiveness of non-small-cell lung cancer to gefitinib. N Engl J Med 350: 2129-2139, 2004

23. Pao W, Miller V, Zakowski M, et al: EGF receptor gene mutations are common in lung cancers from 'never smokers' and are associated with sensitivity of tumors to gefitinib and erlotinib. Proc Natl Acad Sci USA 101: 13306-13311, 2004. 
24. Paez JG, Jänne PA, Lee JC, et al: EGFR mutations in lung cancer: correlation with clinical response to gefitinib therapy. Science 304 : 1497-1500, 2004

25. Giaccone G: Epidermal growth factor receptor inhibitors in the treatment of non-small-cell lung cancer. J Clin Oncol 23: 3235-3242, 2005

26. Han SW, Kim TY, Hwang PG, et al: Predictive and prognostic impact of epidermal growth factor receptor mutation in non-small-cell lung cancer patients treated with gefitinib. J Clin Oncol 23: 2493-2501, 2005.

27. Shigematsu H, Lin L, Takahashi T, et al: Clinical and biological features associated with epidermal growth factor receptor gene mutations in lung cancers. J Natl Cancer Inst 97: 339-346, 2005.

28. Mitsudomi T, Kosaka T, Endoh H, et al: Mutations of the epidermal growth factor receptor gene predict prolonged survival after gefitinib treatment in patients with non-small-cell lung cancer with postoperative recurrence. J Clin Oncol 23: 2513-2520, 2005.

29. Jänne PA, Engelman JA and Johnson BE: Epidermal growth factor receptor mutations in non-small-cell lung cancer: implications for treatment and tumor biology. J Clin Oncol 23: 3227-3234, 2005.

30. Pao W and Miller VA: Epidermal growth factor receptor mutations, small-molecule kinase inhibitors, and non-small-cell lung cancer: current knowledge and future directions. J Clin Oncol 23 : $2556-2568,2005$

31. Imaida K, Yokohira M and Kuno T: Detection of carcinogenic and modifying potentials by test compounds using a mouse lung carcinogenesis bioassay. J Toxicol Pathol 20: 117-123, 2007.

32. Yamakawa K, Kuno T, Hashimoto N, et al: Molecular analysis of carcinogen-induced rodent lung tumors: Involvement of microRNA expression and Kras or Egfr mutations. Mol Med Rep 3: 141-147, 2010.

33. Takeuchi H, Saoo K, Matsuda Y, et al: 8-Methoxypsoralen, a potent human CYP2A6 inhibitor, inhibits lung adenocarcinoma development induced by 4 -(methylnitrosamino) -1-(3-pyridyl)-1-butanone in female A/J mice. Mol Med Rep 2: 585-588, 2009.
34. Renne R, Brix A, Harkema J, et al: Proliferative and nonproliferative lesions of the rat and mouse respiratory tract. Toxicol pathol 37: 5S-73S, 2009.

35. [No authors listed]: The Coronary Drug Project. Findings leading to discontinuation of the $2.5-\mathrm{mg}$ day estrogen group. JAMA 226: 652-657, 1973

36. Chlebowski RT, Schwartz AG, Wakelee H, et al; Women's Health Initiative Investigators: Oestrogen plus progestin and lung cancer in postmenopausal women (Women's Health Initiative trial): a post-hoc analysis of a randomised controlled trial. Lancet 374: 1243-1251, 2009.

37. Slatore CG, Chien JW, Au DH, Satia JA and White E: Lung cancer and hormone replacement therapy: association in the vitamins and lifestyle study. J Clin Oncol 28: 1540-1546, 2010.

38. Chlebowski RT, Anderson GL, Manson JE, et al: Lung cancer among postmenopausal women treated with estrogen alone in the women's health initiative randomized trial. J Natl Cancer Inst 102: 1413-1421, 2010

39. Kitahashi T, Takahashi M, Yamada Y, et al: Occurrence of mutations in the epidermal growth factor receptor gene in X-ray-induced rat lung tumors. Cancer Sci 99: 241-245, 2008.

40. Gealy R, Zhang L, Siegfried JM, Luketich JD and Keohavong P: Comparison of mutations in the p53 and K-ras genes in lung carcinomas from smoking and nonsmoking women. Cancer Epidemiol Biomarkers Prev 8: 297-302, 1999.

41. Hainaut P and Pfeifer GP: Patterns of p53 G $\rightarrow T$ transversions in lung cancers reflect the primary mutagenic signature of DNA-damage by tobacco smoke. Carcinogenesis 22: 367-374, 2001.

42. Zerbe LK, Dwyer-Nield LD, Fritz JM, et al: Inhibition by erlotinib of primary lung adenocarcinoma at an early stage in male mice. Cancer Chemother Pharmacol 62: 605-620, 2008. 